\title{
ANALISIS DE ACTIVIDADES FINANCIERAS
}

\author{
Dr. PASCUAL CHÁVEZACKERMANN (*)
}

\section{RESUMEN}

En otros trabajos hemos descrito la contabilización del capital y la deuda e identificado diversos instrumentos financieros y obligaciones que las corporaciones emplean para financiar sus activos y sus actividades operativas, entre los que se encuentran acciones comunes y preferenciales, bonos pagarés, cuentas por pagar y arrendamientos. Esta vez examinaremos el empleo de la información contable a través del análisis de las actividades financieras, la cual afecta a las relaciones contractuales entre administradores, proveedores de capital, de bienes y servicios. Los administradores tienen la responsabilidad de adoptar decisiones en beneficio de los dueños de la compañía. Al mismo tiempo, deben actuar dentro de las restricciones impuestas por contratos con los acreedores, trabajadores, y otros proveedores de recursos, diseñados para proteger los intereses de las partes contratantes. Por consiguiente, los administradores deben adoptar decisiones que aumenten el valor de una corporación para sus accionistas y que cumplan las obligaciones con los acreedores y otras partes.

Los administradores emplean la información contable para adoptar decisiones financieras. Los inversionistas la emplean para evaluar si las decisiones de la administración concuerdan con el aumento del valor de los accionistas. Los acreedores y otras partes contratantes emplean la información contable para evaluar si las decisiones de la administración satisfacen sus intereses.

La primera parte de este artículo examina los siguientes puntos: la relación entre los atributos de riesgo y ganancia de los instrumentos financieros, el riesgo y la ganancia asociados a los valores, tales como bonos $\mathrm{y}$ acciones que afectan sus precios, las actividades financieras que afectan el riesgo y la ganancia de los valores, y la forma de analizar las actividades financieras para evaluar su efecto en el riesgo y la ganancia.

Los principales temas que trataremos son:

- Efecto de las decisiones financieras en el riesgo y la ganancia.

- Riesgo, ganancia y precio de los instrumentos financieros.

- Uso de la información contable para evaluar las actividades financieras.

Al finalizar el presente estudio, usted será capaz de:

1. Explicar el concepto de estructura del capital.

2. Identificar los efectos de las actividades financieras en los estados financieros.

3. Determinar el efecto del palanqueo financiero en las ganancias de una compañía. 
4. Determinar el efecto del palanqueo financiero en el riesgo de una compañía.

5. Explicar el efecto del palanqueo financiero en el precio de las acciones comunes.

6. Determinar el efecto de las acciones preferenciales en el riesgo y la ganancia.

\section{RIESGO Y GANANCIA}

Las actividades financieras comprenden opciones acerca de cómo obtener efectivo para adquirir otros recursos y pagar servicios. Los administradores pueden elegir entre varios tipos de instrumentos financieros, incluyendo acciones comunes, acciones preferenciales, deuda de corto plazo o largo plazo, y arrendamientos. Los atributos de riesgo y ganancia de dichos instrumentos son variables. Los administradores examinan atributos tales como tasas nominales, cronogramas de reembolso, derechos de canje, provisiones de retiro o rescate, derechos del voto y pago de dividendos. La ilustración muestra acuerdos financieros de varias corporaciones principales e indica la proporción de las finanzas de cada corporación compuesta de diferentes tipos de pasivos y capitales. (Consideramos grandes empresas de nivel mundial).
Los acuerdos financieros varían dependiendo de las actividades operativas y el rendimiento de las compañías. La estructura de capital es la manera que una compañía elige para financiar sus activos y sus actividades operativas. Por ejemplo, la estructura de capital de Mc Donald's incluye una porción grande de deudas de largo plazo. La compañía requiere grandes inversiones en activos de planta, las cuales son financiadas por la deuda de largo plazo. Por el contrario, la estructura de capital de Nike contiene pequeña deuda de largo plazo, pues emplea una gran proporción de acciones preferenciales.

Las compañías de una misma industria pueden tener estructuras de capital diferentes. Por ejemplo, Coca Cola y Mc. Donald's están en la industria alimentaria, pero sus estructuras de capital no son iguales. Lo mismo ocurre con las empresas The Limited y Nike que están en la industria del vestido. La estructura de capital también varía en compañías de diferentes países. En algunos estados como el Japón, es común una gran proporción de deuda financiera, debido a las condiciones económicas y políticas.

La información contable acerca de la estructura de capital de una compañía es

\section{ILUSTRACIÓN $\mathbf{N}^{\circ} \mathbf{1}$}

\section{Acuerdos Financieros como Porcentajes de los Activos Totales de Corporaciones Seleccionadas}

\begin{tabular}{|lc|c|c|c|c|} 
& Coca Cola & The Limited & Mc Donald's & Nike & Walt Disney \\
\hline Pasivos corrientes & $43.0 \%$ & $17.1 \%$ & $9.2 \%$ & $20.7 \%$ & $24.0 \%$ \\
\hline Pasivos a largo plazo & 18.9 & 23.9 & 38.7 & 4.0 & 33.2 \\
\hline Acciones preferenciales & 0.0 & 0.0 & 5.6 & 13.7 & 0.0 \\
\hline Acciones comunes & 12.6 & 7.7 & 2.9 & 5.1 & 7.5 \\
\hline Ganancias retenidas & 25.6 & 51.3 & 43.6 & 56.5 & 35.4 \\
\hline
\end{tabular}

Fuente: Informes Anuales de 1993

Objetivo 1.- Explicar el concepto de estructura del capital. empleada por quienes adoptan decisiones para evaluar el riesgo y la ganancia que es- 
peran de las relaciones contractuales con la compañía. La siguiente sección explica dichos conceptos. nes financieras afectan el rendimiento y el valor de una compañía considere el efecto de las decisiones financieras en los estados

\section{ILUSTRACIÓN No 2}

Efecto de las Decisiones Financieras en los Estados Financieros

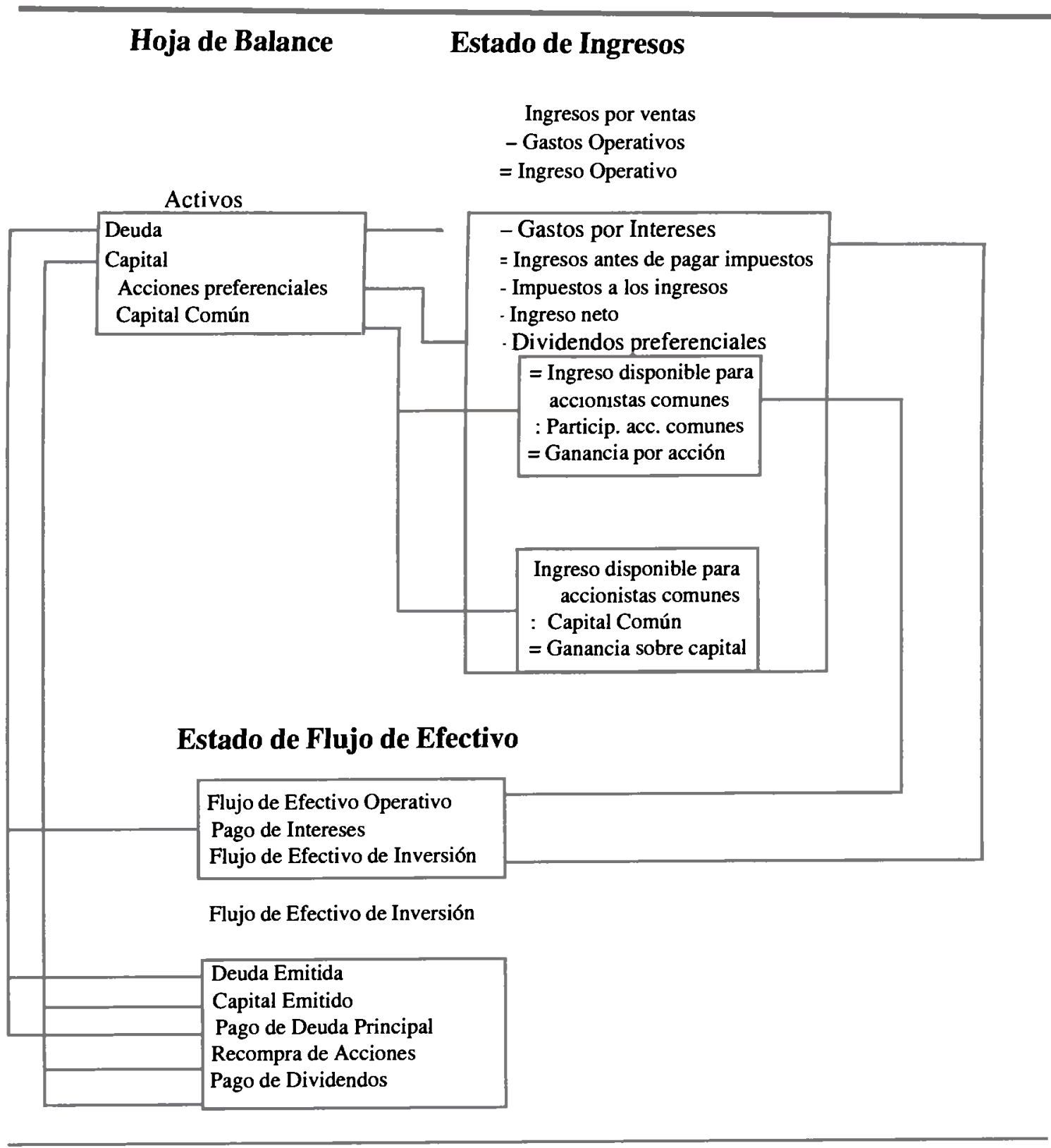

\section{PALANQUEO FINANCIERO}

Objetivo 2.- Identificar los efectos de las actividades financieras en los estados financieros. Para comprender cómo las decisio- financieros de una empresa. La Ilustración $\mathrm{N}^{\circ} 2$ muestra dicho efecto.

Los ingresos por ventas y los gastos operativos son el resultado de actividades 
operativas principalmente. Las partidas que aparecen en el estado de ingresos de una compañía como ingresos operativos no son afectadas por las decisiones financieras. Sin embargo, las partidas que figuran debajo del ingreso operativo si son afectadas, como se muestra en la Ilustración $\mathrm{N}^{\circ} 2$.

Cuando una compañía obtiene un préstamo incurre en el costo de intereses que reduce sus ingresos, pues emplea dinero ajeno. Por consiguiente, el ingreso neto es inferior en la suma del gasto por intereses incurrido durante un periodo. Los préstamos también reducen la cantidad de capital que la compañía debe emitir para financiar sus operaciones. Si una compañía necesita $\$ 10$ millones para adquirir activos y le prestan $\$ 4$ millones, tendrá que emitir sólo $\$ 6$ millones de capital. Si vende sus acciones a $\$ 10$ cada una, deberá vender 600.000 acciones. Si no le prestan dinero, deberá emitir 1 millón de acciones a $\$ 10$ cada una para obtener el financiamiento de $\$ 10$ millones que necesita. Por consiguiente, la ganancia por acción siempre es mayor cuando una compañía obtiene préstamos que cuando emite acciones. El efecto de la deuda sobre las ganancias por acción es conocido como Palanqueo financiero, que es el empleo de deuda para aumentar el rendimiento $y$ las ganancias de una compañía, y es comúnmente medido por la proporción entre la deuda y el capital, en la estructura de capital de la empresa. Una compañía con elevado palanqueo financiero tiene una alta proporción de deuda sobre el capital.

Si asumimos que las ganancias constituyen una medida de las utilidades empleadas por los inversionistas para valorar las acciones de una compañía, mayores ganancias por acción (resultado del empleo de deuda), aumentarán el precio de las acciones de la empresa. Dicho incremento del precio ocurre si el riesgo (tasa de descuento) de la acción no varía. Más adelante examinaremos el efecto de las actividades financieras en el riesgo. Sin embargo, observe que la existencia de deuda en la estructura de capital de una compañía puede mejorar sus ganancias sobre la base de cada acción.

Otra medición que proporciona información similar es la ganancia sobre el capital que es el ingreso disponible para los poseedores de acciones comunes dividida entre el capital en acciones comunes:

Ganancia sobre el capital $=$ (Ingreso Neto - Dividendos Preferenciales) / Capital en Acciones Comunes

En forma similar que la ganancia por acción (GPA), la ganancia sobre el capital (GSC) es una medida del rendimiento relativo de la compañía luego de considerar el efecto de las decisiones financieras. Una ventaja de la GCS sobre la GPA es que la GSC puede ser comparada entre compañías. Por ejemplo, se compara con el tiempo si el número de acciones no varía y con otras mediciones expresadas sobre la base de cada acción, como ocurre con el precio de las acciones. Sin embargo, y con otras mediciones es difícil compararla entre compañías porque éstas tienen diferente número de acciones pendientes. Por lo tanto, si la Compañía A consigna ganancias del $\$ 2$ por acción, y la Compañía $\mathrm{B}$ de $\$ 3$ por acción, no podemos determinar que la Compañía $B$ ha tenido mejor rendimiento que la Compañía A. Sin embargo, si la ganancia sobre el capital de la Compañía B es $25 \%$ y de la Compañía A $15 \%$, podemos tener certeza de que la Compañía $B$ ha obtenido mejor rendimiento que la Compañía $\mathrm{A}$.

La emisión de deuda y capital también afectan el flujo de efectivo. La compañía obtiene efectivo cuando emite deuda y capital, pero entrega efectivo cuando el principal, los intereses y los dividendos son 
pagados. Asimismo, entrega efectivo cuando recompra acciones. Por consiguiente, además de evaluar el efecto de las decisiones financieras sobre las ganancias de una compañía, es importante evaluar su efecto en el flujo de efectivo. En particular, es indispensable determinar si una compañía puede generar suficiente efectivo para cumplir sus obligaciones y satisfacer sus otras necesidades. Más adelante volveremos al tema del flujo de efectivo. Primero, considere cómo las actividades financieras afectan los ganancias de los accionistas.

\section{EFECTO DEL PALANQUEO FINANCIERO SOBRE LAS GANANCIAS}

Objetivo 3.- Determinar el efecto del palanqueo sobre las ganancias de la compañía.
Capital y la Compañía Deuda, poseen activos por $\$ 1.000$ y obtienen $\$ 250$ de ingreso operativo.

Por lo tanto, las dos compañías son iguales, excepto en la financiación. La Compañía Capital emplea 70\% de capital en su estructura de capital, mientras que la Compañía Deuda emplea $70 \%$ de deuda. El resultado de esta diferencia es que la Compañía Capital incurre en menor gasto por intereses ( $\$ 30$ en relación a $\$ 70$ de la Compañía Deuda). La tasa de impuestos para ambas compañías es de $30 \%$. Por consiguiente, el ingreso neto y disponible de la Compañía Capital es mayor que el de la Compañía Deuda (\$ 154 en comparación a \$126). Sin embargo, la Compañía Capital emplea mayor número de acciones para financiar sus operaciones que la Compañía

\section{ILUSTRACIÓN No 3}

\section{Efecto del Palanqueo Financiero sobre la Ganancia sobre el Capital}

\begin{tabular}{|c|c|c|c|c|c|c|}
\hline Activos & $\begin{array}{c}\text { Cía. } \\
\text { Capital } \\
1.000\end{array}$ & $\begin{array}{c}\text { Cía. } \\
\text { Deuda } \\
1.000\end{array}$ & $\begin{array}{c}\text { Cía. } \\
\text { Capital }\end{array}$ & \multicolumn{3}{|c|}{$\begin{array}{l}\text { Cía. } \\
\text { Deuda }\end{array}$} \\
\hline Deuda & 300 & $700 \rightarrow$ & $30 \%$ & & $30 \%$ & \\
\hline Capital & 700 & 300 & & & & \\
\hline Acciones & 100 & $50 \rightarrow$ & $70 \%$ & & & \\
\hline Ingreso operativo & 250 & 250 & & & -70 & \\
\hline Gasto por intereses & 30 & $70 \longrightarrow$ & -30 & & & \\
\hline Ingresos antes de pag. impuestos & 220 & 180 & & & & \\
\hline Impuesto a los ingresos & 66 & 54 & 154 & 700 & 126 & 300 \\
\hline Ingreso neto & 154 & $126 \uparrow$ & & & & \\
\hline Dividendos preferenciales & 0 & $0 \uparrow$ & & & & \\
\hline Ingresos disp. para acc. comunes & 154 & 126 & & & & \\
\hline Ganancia por acción & 1,54 & $2,52 \ldots$ & & & & \\
\hline Ganancia sobre el capital & $22 \%$ & $42 \%$ & & lanci & bre el c & ital \\
\hline
\end{tabular}

Para ilustrar el efecto de las decisiones financieras sobre el rendimiento, considere los resultados de las dos compañías de la Ilustración $\mathrm{N}^{\circ} 3$. Ambas, la Compañía
Deuda. Por lo tanto, en relación al monto del capital invertido, la Compañía Capital obtiene menores ganancias (22\%) que la Compañía Deuda (42\%). 
En este análisis, dos factores principales son importantes. Primero, la deuda trae consigo gasto por intereses que reduce las ganancias. Segundo, también disminuye la cantidad de capital que la compañía debe emitir. Por ello, la ganancia sobre el capital (o ganancias por acción), a menudo es mayor cuando la deuda es incluida en la estructura de capital de la compañía. La deuda puede ser beneficiosa para los accionistas de una compañía.

\section{EFECTO DEL PALANQUEO} FINANCIERO EN EL RIESGO

Objetivo 4.- Estudiar el efecto del palanqueo financiero en el riesgo de una compañía.
La ilustración muestra tres tipos de condiciones para las compañías Capital y Deuda que corresponden a un año malo, uno normal y otro bueno. En un año malo, el ingreso operativo de ambas compañías es \$ 50. En un año normal es de $\$ 250$, y en un buen año, $\$ 450$. Sin embargo, observe que los ingresos y la ganancia sobre el capital no son los mismos para ambas empresas. La compañía Capital incurre en menores intereses que la compañía Deuda. Dicho gasto ocurre tanto si la compañía tiene un año malo, normal, o bueno. Si el año es malo, ambas instituciones consignan menor ingreso que la compañía Capital. Informa una pérdida (y una ganancia sobre el capital GSC negativa), porque su gasto por intereses es mayor que su ingreso operativo. Si el año es

\section{ILUSTRACIÓN No 4}

\section{Efecto del Palanqueo Financiero en el Riesgo}

\begin{tabular}{lccc|rrr} 
& \multicolumn{4}{c|}{ Compañía Capital } & \multicolumn{3}{c}{ Compañía Deuda } \\
\hline & $\begin{array}{c}\text { Año } \\
\text { Malo }\end{array}$ & $\begin{array}{c}\text { Año } \\
\text { Normal }\end{array}$ & $\begin{array}{c}\text { Año } \\
\text { Bueno }\end{array}$ & $\begin{array}{c}\text { Año } \\
\text { Malo }\end{array}$ & $\begin{array}{c}\text { Año } \\
\text { Normal }\end{array}$ & $\begin{array}{c}\text { Año } \\
\text { Bueno }\end{array}$ \\
\hline Activos & $\$ 1.000$ & $\$ 1.000$ & $\$ 1.000$ & $\$ 1.000$ & $\$ 1.000$ & $\$ 1.000$ \\
Deuda & 300 & 300 & 300 & 700 & 700 & 700 \\
Capital & 700 & 700 & 700 & 300 & 300 & 300 \\
Ingreso operativo & 50 & 250 & 450 & 50 & 250 & 450 \\
Gasto por intereses & 30 & 30 & 30 & 70 & 70 & 70 \\
Ingreso antes de pagar imp. & 20 & 220 & 420 & -20 & 180 & 380 \\
Impuesto a los ingresos & 6 & 66 & 126 & -6 & 54 & 114 \\
Ingreso Neto & 14 & 154 & 294 & -14 & 126 & 266 \\
Ganancia sobre el capital & $2,0 \%$ & $22 \%$ & $42 \%$ & $-4,7 \%$ & $42,0 \%$ & $88,7 \%$ \\
& & & & & & \\
\hline
\end{tabular}

Es posible que el empleo de una deuda aumente la ganancia sobre el capital de una compañía. Sin embargo, no está exento de costo. Considere el efecto del palanqueo financiero en la variabilidad de los ingresos y las ganancias sobre el capital descrito en la Ilustración $\mathrm{N}^{\circ} 4$. bueno, ambas compañías consignan altos ingresos. La compañía Deuda consigna mayor ganancia sobre el capital que la compañía Capital, debido a su menor cantidad de capital.

Por consiguiente, en años buenos ( $y$ en años normales), la compañía Deuda obtiene 
mejor rendimiento que la compañía Capital. Pero en los años malos, la compañía Capital tiene mejor rendimiento que la compañía Deuda. El gráfico de la Ilustración $\mathrm{N}^{\circ} 4$ muestra la relación entre el ingreso operativo y la ganancia sobre el capital de las dos compañías. Al aumentar el ingreso operativo, la ganancia sobre el capital crece en ambas empresas. Sin embargo, aumenta más rápidamente para la compañía Deuda que para la compañía Capital. El declive de la línea que indica la relación es más pronunciado para la compañía Deuda. Esto significa que la compañía Deuda es más riesgosa que la compañía Capital.

Objetivo 5.- Explicar el efectodel palanqueo financiero en el precio de las acciones comunes.

Los ingresos y las ganancias sobre el capital de la compañía Deuda son más volátiles que los de la compañía Capital. Esto significa que los ingresos y las ganancias sobre el capital son menos seguros. Por ello, de efectivo por sus inversiones en la compañía Deuda, también piden ganancias más altas para compensar el mayor riesgo, como se muestra en la Ilustración $N^{\circ} 5$.

$\mathrm{Al}$ aumentar el palanqueo financiero de una compañía,(deuda/capital), las ganancias previstas probablemente se incrementen. $\mathrm{El}$ riesgo también crece. Por lo tanto, el efecto sobre el precio de las acciones no puede ser determinado sin información adicional. Durante los periodos en los cuales los inversionistas esperan que el ingreso operativo de la compañía aumente, probablemente percibirán que la deuda es beneficiosa debido a las mayores ganancias previstas. Sin embargo, si estiman que el ingreso operativo será bajo, percibirán que la deuda no es beneficiosa.

El palanqueo financiero magnifica el rendimiento de una compañía. Si el ingreso operativo es alto, el palanqueo financiero conduce a altas ganancias sobre el capital.

\section{ILUSTRACIÓN No 5}

\section{Efecto de Palanqueo Financiero en el Riesgo y la Ganancia}

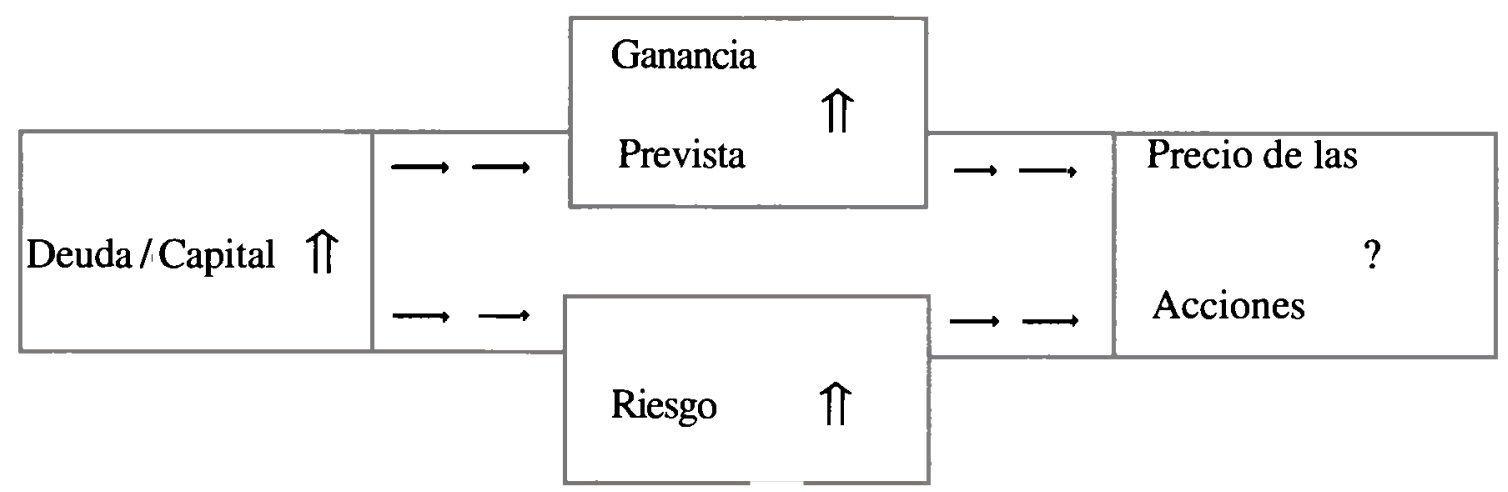

deberíamos suponer que los inversionistas exigirán mayor tasa de utilidad por sus inversiones en la compañía Deuda que en la compañía Capital. En consecuencia, aunque los inversionistas pueden recibir mayor flujo
Si es bajo, conduce a bajas ganancias sobre el capital. Es posible que el palanqueo financiero tenga efecto similar en el precio de las acciones. Cuando el ingreso operativo es alto, los precios de las compañías con alto 
palanqueo financiero serán más elevados que aquellos de compañías con bajo palanqueo y similares en otros aspectos. Cuando el ingreso operativo es bajo, los precios de las compañías con alto palanqueo serán menores que aquellos de compañías similares con bajo palanqueo.

\section{EFECTO DE LAS ACCIONES PREFERENCIALES EN EL RIESGO Y LA GANANCIA}

Objetivo 6.- Determinar el efecto de las acciones preferenciales en el riesgo y la ganancia.

La acción preferencial es similar a la deuda en que el emisor promete pagar una ganancia al comprador. El monto de la ganancia usualmente es fijado según una tasa acordada cuando la acción preferencial es emitida. El monto pagado a los accionistas preferenciales reduce el efectivo disponible para el pago de dividendos a los accionistas comunes o para reinvertir en la compañía para crear valor futuro adicional. También, como la deuda, el empleo de acciones preferenciales como fuente de financiamiento, reduce el monto de capital común necesario. Por ello, las acciones preferenciales producen un efecto de palanqueo similar a la deuda. Por ejemplo, suponga que la compañía Capital desea obtener nuevo financiamiento para expansión de planta. $\mathrm{La}$ compañía necesita $\$ 400$ para la expansión. ¿Cuál sería el efecto de usar acciones preferenciales para el nuevo financiamiento? Suponga que la compañía puede emitir acciones preferenciales a $\$ 1$ a la par que debería pagar una ganancia de $8 \%$. La Ilustración $\mathrm{N}^{\circ} 6$ describe los resultados de la decisión financiera.

Antes del nuevo financiamiento, la compañía Capital $\$ 1.000$ tenía en activos financiados completamente por acciones comunes. El nuevo financiamiento requerirá

\section{ILUSTRACIÓN No 6}

Efecto de las Acciones Preferenciales en la Ganancia

\begin{tabular}{|l|c|c|}
\hline & $\begin{array}{c}\text { Emite Acciones } \\
\text { Preferenciales }\end{array}$ & $\begin{array}{c}\text { Emite Acciones } \\
\text { Comunes }\end{array}$ \\
\hline Activos existentes & 1.000 & 1.000 \\
Nuevos activos & 400 & 400 \\
Capital existente & 1.000 & 1.000 \\
Nuevas acciones preferenciales & 400 & 0 \\
Nuevas acciones comunes & 0 & 400 \\
Ingreso operativo & 300 & 300 \\
Gasto por intereses & 0 & 0 \\
Ingreso antes de pagar impuestos & 300 & 300 \\
Impuesto a los ingresos & 90 & 90 \\
Ingreso neto & 210 & 210 \\
Dividendos preferenciales & 32 & 0 \\
Ingreso disponible para accionistas comunes & 178 & 210 \\
Capital común & 1.000 & 1.400 \\
Ganancia sobre el capital & 17,8 & $15 \%$ \\
\hline
\end{tabular}


de $\$ 400$ de acciones preferenciales o de acciones comunes adicionales por el mismo monto. Supondremos que la compañía espera un ingreso operativo de $\$ 300$ después de la expansión de su planta. Ello podría ocurrir ya sea que la compañía emita acciones preferenciales o comunes. No se incurrirá en gasto por intereses porque la compañía no tiene deudas. Se supone que la tasa de impuestos será $30 \%$. Por lo tanto, la compañía obtendrá $\$ 210$ de ingreso neto sin importar la alternativa que emplee. Sin embargo, si emite acciones preferenciales, la compañía pagará dividendos de $\$ 32$ $(8 \% \times \$ 400)$ a los accionistas preferenciales.

Dicho pago deja \$ 178 disponibles para los accionistas comunes (por dividendos y reinversiones). También, la emisión de acciones preferenciales reduce la cantidad de acciones comunes pendientes que existían si se emitiesen acciones comunes. Como resultado de esta operación, el ingreso sobre el capital común es más elevado si se emiten acciones preferenciales que si se emiten acciones comunes. Las acciones preferenciales producen un efecto de palanqueo financiero similar al de la deuda.

La emisión de acciones preferenciales también aumenta el riesgo de una compañía, en forma similar al efecto de la deuda. Los dividendos preferenciales usualmente deben ser pagados ya sea en el año corriente o en algún año futuro. Si el ingreso neto de una compañía es bajo, las acciones preferenciales reducirán la ganancia sobre el capital porque los dividendos reducen aún más el ingreso disponible para los accionistas comunes. $\mathrm{Si}$ el ingreso neto es alto, las acciones preferenciales aumentarán la ganancia sobre el capital, porque el monto de capital común será menor que si se emplearan acciones comunes para financiar a la compañía. Por lo tanto, las acciones preferenciales aumentan el efecto de las variaciones del ingreso neto en la ganancia sobre el capital.
Una desventaja de las acciones preferenciales con respecto a la deuda financiera es que los dividendos no son deducibles para fines tributarios. Por consiguiente, una compañía no reduce su pago de impuestos como cuando paga intereses. Si una compañía paga $\$ 1$ millón en dividendos (preferenciales o comunes), su egreso de efectivo es $\$ 1$ millón. Sin embargo, si paga $\$ 1$ millón de intereses y su tasa de impuestos es $30 \%$, su egreso de efectivo actual es sólo $\$ 700.000$ debido al ahorro de impuestos. Por otro lado, los accionistas preferenciales, a diferencia de los acreedores, no pueden forzar la bancarrota de una compañía. Las acciones preferenciales tienen algunas características de la deuda y otras del capital. Son una fuente alternativa de financiamiento que atrae a algunas compañías e inversionistas. 